\title{
Correlation Features of Microcirculation Parameters in Children with Type 1 Diabetes Mellitus and Chronic Tonsillitis
}

\author{
Iurii Gavrylenko ${ }^{1}$, Andrei Laiko ${ }^{1}$, Inga Mityuryayeva ${ }^{2}$, Anna Hnyloskurenko $^{2}$ \\ ${ }^{I}$ Department Of Pediatric Otorhinolaryngology, Audiology And Phoniatrics / Shupyk National Medical Academy \\ Of Post-Graduate Education, Ukraine \\ ${ }^{2}$ Department Of Pediatrics №4/ Bogomoltsa National Medical University, Ukraine
}

\begin{abstract}
This paper deals with the study of disorders of the microvasculature in children with type 1 diabetes mellitus (DM-1) and chronic tonsillitis (HT). Clinical and laboratory examination of 93 children with DM-1, aged 5 to 17 was conducted, as well as the correlation between the indicators of bulbar microscopy and mucosa microrhinoscopy of the nasal septum in this group of patients with the presence of HT, the curvature of the nasal septum (CNS) and the recurrent epistaxis (RE) was analyzed. According to the results of the correlation analysis in children with DM-1there was established a direct relationship between the presence of chronic ENT-pathology and the presence of vascular glomeruli $(r=0,529, p<0.05)$, as well as changes in the capillary network structure $(r=0,332, p<0,05)$ according to bulbar microscopy. These findings point at a significant lesion of the microvasculature in children with DM-1on the background of ENT pathology, especially in the presence of chronic tonsillitis and recurrent epistaxis.
\end{abstract}

Keywords: type 1 diabetes, children, microcirculation, bulbar microscopy, chronic tonsillitis.

\section{Introduction}

Currently, diabetes mellitus has become epidemic, as one of the most common causes of disability and mortality. Today in Ukraine there are about 1 million of diabetic patients, of which 10-15\% suffer from diabetes mellitus type 1 (DM- 1), including 4.6\% - children [1]. DM- 1 is a disease which is based on the all types of metabolism disorder with the gradual development of a universal microangiopathy. The development of pathological changes in microvasculature, including the retina; patients with DM- 1 suffer from the most severe vascular complications [2].Until recently, microvascular changes in children and adolescents with DM-1 were considered to be rare. However, conducted epidemiological studies revealed early development of microangiopathy in younger children and adolescents. The progress of the disease and poor glycemic control lead to severe disturbance of capillary blood flow [3]. Functional disorders of the vessels of microcirculatory channel are accompanied by restructuring and, as a rule, lead to hyperplasia of lymphoid tissue [4]. According to the literature evidence, the pathology of upper respiratory tract in adults occurs in 59\% of patients with diabetes [5], but until now the issue of pathology of upper respiratory tract in children and adolescents with DM- 1 and features of microcirculation disturbance in such patients have still been poorly investigated [6].

To study the correlation of parameters of microcirculatory channel of the nasal septum mucous membrane and bulbar microscopy in children with type 1 diabetes mellitus on a background of chronic tonsilitis.

\section{Materialsandmethods}

During the period 2014-2015 years we examined 93 children with DM- 1, who were treated in the endocrinology department №6 in Kyiv and the National Children's Specialized Hospital "OHMATDET". The age of patients was from 5 to 17 years. Among all examined patients $37(39.7 \%)$ - girls, and $56(60.3 \%)$ - boys. All patients received general and otolaryngology clinical examination, under which the two groups were identified. The first group (main) included $70(75.3 \%)$ children with diabetes mellitus, with found chronic diseases of upper respiratory tract. The second group (control) consisted of 23 (24.7\%) children, without otolaryngology pathology. Evaluation of the microcirculatory channel state was performed by using nail bed capillaroscopy. The state of the microcirculation of bulbar conjunctiva was evaluated by using a slit lamp with subsequent photo registration. Statistical analysis of the data by descriptive statistics method and correlation analysis were performed by using SPSS 17,0 program. The result at $\mathrm{p}<0.05$ was considered to be a significant difference. 


\section{Research results}

To the main group patients with pathology of upper respiratory tract in the form of chronic tonsillitis (HT) - 34 (48.5\%), curvature of the nasal septum (CNS) - 16 (22.9\%), recurrent nose bleeding (RNB, Epistaxis) 10 (14.3\%) and $10(14.3 \%)$ patients with nosebleeds found in conjunction with the CNS were selected. (Figure 1)

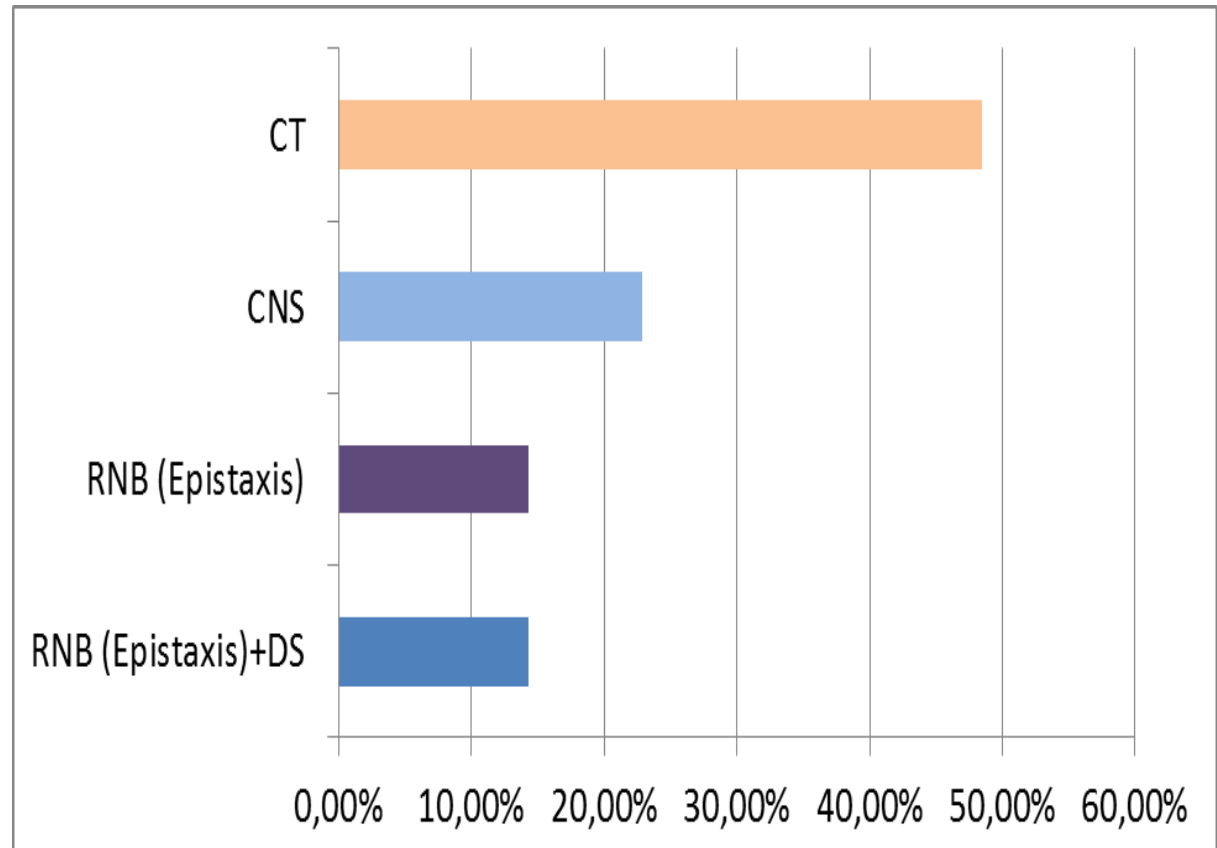

Figure 1.Structure of ENT pathology in patients with DM- 1 (main group).

In assessing of medical history data the average duration of DM-1 disease in the main group was $3,68 \pm$ 0,05 years, in the control group - 2,8 $\pm 0,2$ years $(\mathrm{p}<0.05)$.

Among patients with DM-1 of the main group on the background of ENT -pathology the number of children with high risk for life glycemic control far outweighed $(61.4 \%$ vs. $34.8 \%$ of children in the control group) (p < $<.05)$. The difference between groups was significant $(\mathrm{p}<0.05)$.

Evaluation of glycemic control in both groups is presented in Table 1.

Table 1.Classification of patients with DM- 1 in the main and control groups depending on the level of glycemic control.

\begin{tabular}{|l|l|l|}
\hline Glycemiccontrol & $\begin{array}{l}\text { Main group } \\
(\mathrm{n}=70)\end{array}$ & $\begin{array}{l}\text { Control group } \\
(\mathrm{n}=23)\end{array}$ \\
\hline Optimal & $5(7,2 \%)^{*}$ & $13(56,5 \%)$ \\
\hline Suboptimal & $22(31,4 \%)$ & $2(8,7 \%)$ \\
\hline With high risk for life & $43(61,4 \%)^{*}$ & $8(34,8 \%)$ \\
\hline
\end{tabular}

Note: * - $p<0.05$ when compared the patients of both groups

When we look at the main group, the tendency to the increase of the pathology of upper respiratory tract with deterioration of glycemic control is observed (Figure 2). 


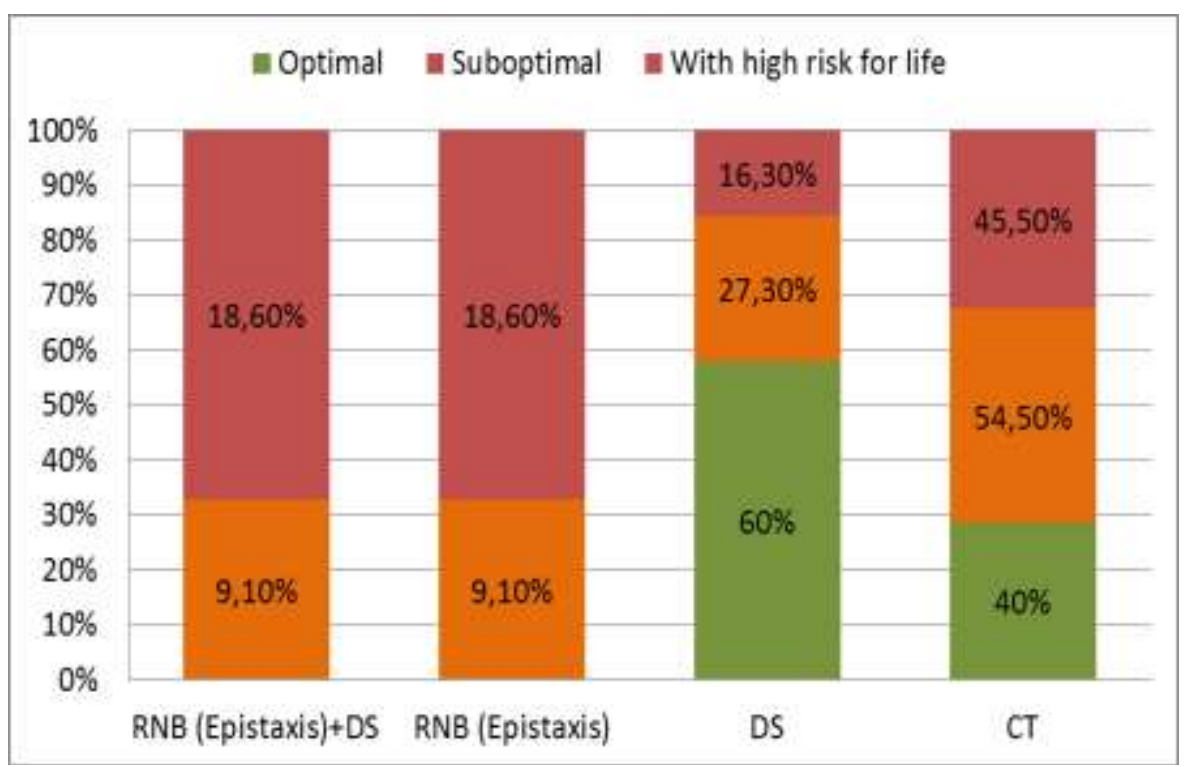

Figure 2. Classification of patients with ENT - pathology on the level of glycemic control

With a detailed evaluation of the microcirculatory channel of nasal cavity in patients from the main group with DM- 1 depending on the chronic pathology of upper respiratory tract various diagnostic parameters were obtained (Figure 3).

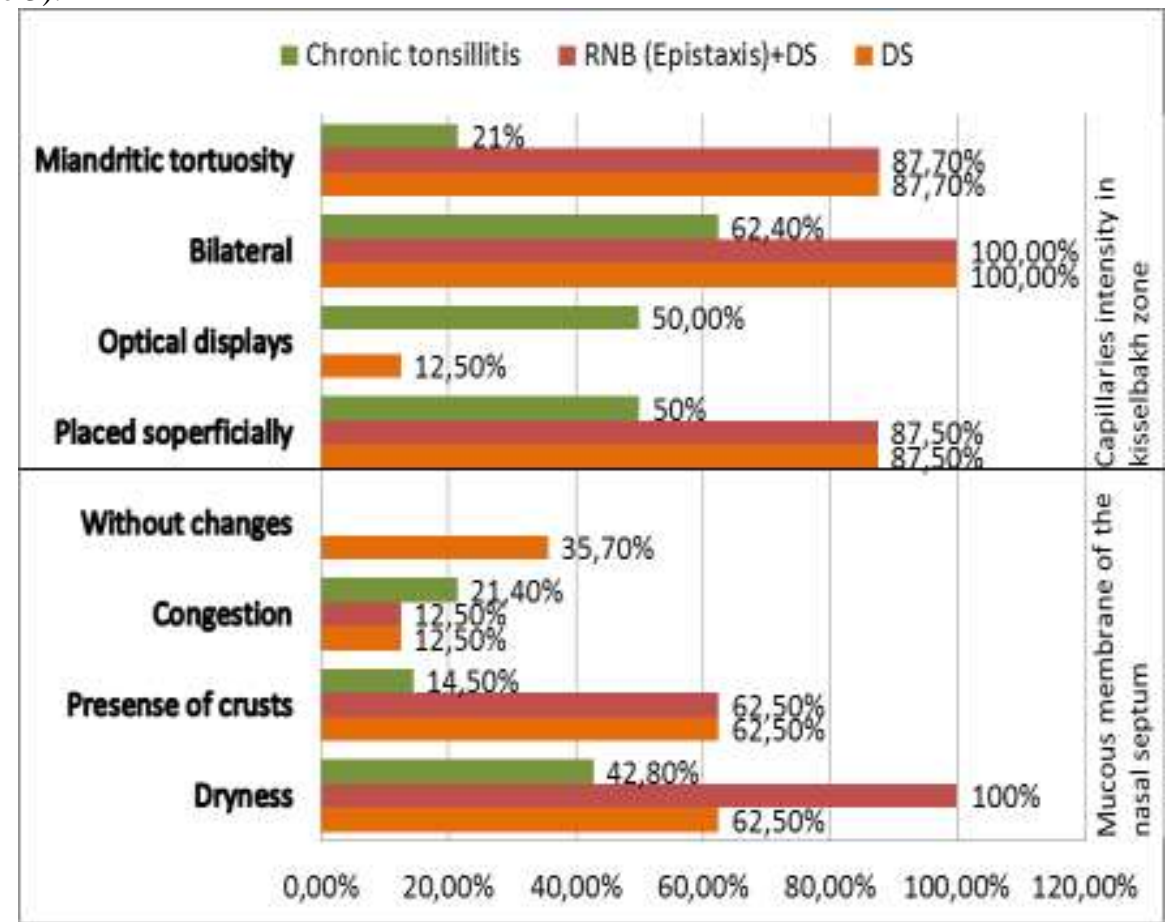

Note: * - p <0.05 when compared different groups of patients

Figure 3.Parameters of microcirculatory channel of the nasal septum mucous in patients of the main group.

Thus, the received results indicate the interrelation of otolaryngology chronic pathology with severe microcirculation disturbance in patients with type 1 diabetes.

According to the correlation analysis a direct correlation between the presence of chronic ENT pathology and the presence of vascular glomeruli $(r=0,529, \mathrm{p}<0.05)$, as well as with changes in capillary meshwork $(r=0,332, p$ $<0.05)$ was established according to bulbar microscopy . 
Correlation Features of Microcirculation Parameters In Children with Type 1 Diabetes Mellitus....

Thus, a direct correlation between indicators of nasal septum mucosa microscopy and venulasacculation was established according to bulbar microscopy. Also, correlation between the presence of abnormal blood vessels and the presence of vascular glomeruli and venulasacculation, besides, indicator of miandritic tortuosity of the capillaries had a direct correlation with the presence of vascular glomeruli, uneven caliber vessels

\section{Conclusion}

On conducting of correlation analysis a direct correlation between the level of glycemic control and the presence of abnormal vessels of the nasal septum mucous membrane in the Kisselbach zone, diagnosed chronic ENT pathology and the presence of vascular glomeruli, and also capillary meshwork changes according to bulbar microscopy was revealed in patients with diabetes mellitus type 1, which confirms the influence of DM -1 disease on disturbance of microcirculatory channel state. It was also established that on chronic pathology of ENT - organs in children with DM-1, infectious and inflammatory changes of the mucosa nasal membrane lead to disturbance of the peripheral trophic of its tissues, and impair general pathological shift of microcirculatory channel which require timely and effective treatment. These data suggest the need for early and timely diagnosis of the upper respiratory tract pathology in children with type 1 diabetes mellitus and adequate correction of microcirculation changes.

\section{REFERENCES}

[1]. A. Bezdetko, A. Gorbacheva, Epidemiology and incidence of diabetes and diabetic retinopathy, International Journal of Endocrinology, 4 (6), 2006, 76-80.

[2]. O. Bogoyavlenskaya, V. Oslopov, Investigation of the microcirculation state in hypertension, Kardiologiya, 5 (10), $2010,20-25$.

[3]. G. Scardina, A. Cacioppo, T. Pisano, In vivo evaluation of labial microcirculation in diabetics: a comparision of healthy subjects, Panminevra Med, 53, 2011, 81-85.

[4]. M. Boisvert, K. Koski, D. Burns, C. Skinner, Prediction of gestational diabetes mellitus based on an analysis of amniotic fluid by capillary electrophoresis, Biomarkers in Medicine, 6 (5), 2012, 645-653.

[5]. A. Gurov, E. Biryukova, M. Yushkina, Modern problems of diagnosis and treatment of inflammatory diseases of upper respiratory tract in patients with diabetes, Vestnikotorinolaringologii, 2, 2011, 76-79.

[6]. Iu. Gavrylenko, Clinical and epidemiological aspects of ENT diseases in children with diabetes mellitus type 1, International scientific journal, Pediatrics, Eastern Europe, 4 (12), 2015, 68-75. 